\section{ILLUSTRATIONS OF THE DIAGNOSTIC VALUE OF AGGLUTININ DETERMINA- TIONS IN INOCULATED INDIVIDUALS.}

BY E. W. AINLEY WALKER, M.A., D.M. OXoN., CAPTAIN O.T.C., ATTACHED 3RD SOUTHERN GENERAL HOSPITAL; FELLOW
AND TUTOR OF UNIVERSTY COLLEGE AND LECHURER IN PATHOLOGY IN THE UNIVERSITY OF OXFORD.

(A Report to the Medioal Researoh Committee. From the Department of Pathology, Oxford.)

IN the blood of individuals inoculated against typhoid fever there are present certain quantities of agglutinins for B. typhosus. The amount of these agglutinins is so remarkably diverse in different individuals, even when they have received identical doses of the one and same vaccine and are examined after the same lapse of time, ${ }^{1}$ that it is quite impossible to arrive at any kind of figure which could be taken to represent their probable value in any given individual at any given interval after his inoculation. Accordingly it is impossible to decide on making a determination of agglutinin titre whether the agglutination observed is wholly the result of past inoculation, or whether it contains evidence of superadded active infection. Recognition of these facts a year and more ago led a number of experienced pathologists to declare that the Widal reaction failed to afford information of diagnostic value as regards typhoid infection in typhoid-inoculated individuals. Where there was danger that this fact might not be recognised so speedily, it was abundantly brought home by clinical experience of "the failure of the Widal test due to inoculation." 2

Under these circumstances Dreyer, Ainley Walker, and Gibson ${ }^{3}$ drew attention to the fact that in these inoculated individuals a diagnosis could still readily be made by means of the agglutinin reaction if the agglutination test were repeated several times at short intervals in order to ascertain whether the titre of agglutination showed marked changes affording evidence of an immunity reaction such as is known to accompany active infection. But for this purpose it was necessary to employ an accurately quantitative method of determination, and one whose results were always comparable inter se. It was therefore proposed to make use of the method published by Dreyer in 1906 (rendered more accessible to British pathologists in $1909^{4}$ ), which had for years proved eminently satisfactory, and was then (as it still remains to-day) the only published method which adequately fulfils all the requirements. And it was shown that where this method was used in the examination of individuals inoculated against typhoid fever the agglutinin reaction still afforded the simplest and the most reliable method of diagnosis in suspected cases of typhoid infection.

Now it is obvious that what could thus be done in regard to the diagnosis of typhoid fever in individuals inoculated against B. typhosus can also be done for paratyphoid A infection in persons inoculated against $B$. paratyphosus $\mathbf{A}$ and for paratyphoid $B$ infection in persons inoculated against B. paratyphosus $\mathbf{B}$, probably also for enteric infections (typhoid and paratyphoid), generally in persons inoculated against all three micro-organisms. Yet since the introduction of inoculation with triple vaccine (B. typhosus, B. paratyphosus A, and B. paratyphosus B) the old notion that the agglutinin reaction can no longer be employed as a ready diagnostic instrument shows a tendency to reassert itself. Unfortunately it even makes its reappearance under the protection of some pathologists who have had ample opportunity of seeing evidence to the contrary. This would be a matter of the less urgent importance were there alternative methods of diagnosis at disposal which offered a reasonably equal probability of success The facts, however, are quite the reverse. Failing the agglutinin reaction, recourse must necessarily be had to methods of blood cultures and to cultivation from the stools and urine. And all these methods require not only extensive preparation and expenditure of material, but very considerable skill and experience. They are laborious, so that the worker can at best not handle more than a fraction of the number of cases that he could easily examine by agglutination tests. They are complicated and cannot invariably be carried out under active service conditions. Worse than all, even though repeated examinations are carried out, they very often fail entirely. The infecting micro-organism frequently refuses to be isolated, and evidence justifies the statement that quite half the cases will remain undetected where these methods are exclusively relied upon.

Now it is obvious that under present conditions no one would willingly or consciously assume the grave responsibility entailed in failure to detect cases of typhoid or paratyphoid fever which are capable of being readily detected. Yet this is what must necessarily occur, to the gross prejudice of general hygiene and efficiency, if only those cases of " enteric fever" are detected which yield a positive result by culture methods. On the other hand, the agglutination test, when suitably carried out and appropriately repeated, can be made to yield a uniformly accurate diagnosis in cases examined during active infection. It has been shown by Dreyer ${ }^{5}$ and others to give correct results over long series of cases in which the diagnosis was rendered absolute by tine successful cultivation of the infecting micro-organism from blood, stools, or urine. It may, therefore, safely be presumed also to give reliable results in cases where the infective agent itself has not been isolated and identified.

It has been suggested that needful assistance would be given to others in the interpretation of results obtained in such agglutination tests by the publication of a series of illustrative charts from actual cases with brief explanatory notes.

\section{Notes and Illustrations.}

In interpreting the results of agglutination tests attention is directed to the known form of the immunity curve of agglutinin production resulting from a natural (or artificial) infection. The general outline of such curves is now familiar. They were first traced by accurately quantitative methods in typhoid fever by Madsen and Jörgensen, ${ }^{6}$ and subsequently with still greater certainty by Dreyer and Schroeder, ${ }^{7}$ employing Dreyer's standardised agglutinable cultures. Similar curves were also given by Dreyer and Ainley Walker, ${ }^{3}$ both for specific and for non-specific stimulation of agglutinin formation under experimental conditions.

A good example of an agglutinin curve is shown in Chart I. which is here reproduced from a previous article. ${ }^{3}$ The broken line represents the course of agglutinin formation in a case of typhoid fever-the curve of immunity reaction for agglutinins -and set over against it in thick lines are observations on three cases occurring in typhoid inoculated individuals designated by the signs $\alpha, \beta$, and $\gamma$ respectively. The first two of these cases were cases of active paratyphoid $B$ fever, the third case was " not enteric."

Before proceeding to the actual description of illustrative curves * it will be of advantage to collect together certain data on which diagnosis proceeds. Most of these data have already appeared in the Directions for Use of Standardised Agglutinable Cultures issued on behalf of the Medical Research Committee or have been published elsewhere by Dreyer and his co-workers. Their original statements have received additions and extensions from time to time as knowledge and experience have accumulated. Doubtless these statements may be subject to some further elaboration or to some qualification in the future, but the main outlines have been firmly established from an early date and have proved themselves to be reliable in the opinion of other workers. ${ }^{9}$

1. In non-inoculated persons who have never had enteric fever $\uparrow$ agglutination in a dilution of 1 in 25 ( 1 in 10 paratyphoid $\mathrm{A}^{5}$ ) justifies a strong suspicion of active infection. But the test must be repeated in the course of a few days to ascertain whether there is any change in the titre of agglutination. Marked agglutination in dilution of 1 in 50 or more is (nearly always) diagnostic of active infection. A non-inoculated " carrier," in whom infection is inactive, will normally show no important change in the titre of his serum on repeated examination at short intervals.

2. Inoculated persons, if quite recently inoculated, will usually show a high titre of specific agglutination. The curve in Chart 1, from a case of typhoid fever, serves equally well to show the general form of the curve of agglutinin

- Detailed agglutinin curves for enteric fevers in inoculated individuals were first worked out by Dreyer a year or more ago from daily observations in a number of patients. But the data were unfortunately in great part destroyed by fire.

$\dagger$ Here and elsewhere the term enteric fever includes both typhoid and paratyphoid infection. 
formation following inoculation. A rapid rise in titre (for each bacillus in the case of triple vaccine) sets in within a few days of inoculation. A maximum is reached, followed by a fall at first rapid, but subsequently becoming very slow, so that a relatively high titre may be maintained for a long period (even for years). During this period examinations made at intervals of a few days give practically identical readings.

3. When an inooulated individual suffers from an attack of enteric fever his titre of agglutination for the bacillus concerned (typhosus, paratyphosus $A$; or paratyphosus B) will exhibit the usual rise and subsequent regular fall seen in non-inoculated subjects (Chart 1), but starting from and returning towards the higher base line of inoculated persons. Meanwhile one of three things may occur as regards the agglutinin titres of his serum for other members of the group against which he has been protectively inoculated:-

(a) No appreciable rise may occur in the titres.

(b) A relatively slight rise may occur, followed by a fall towards their former level.

(o) A marked rise may occur approximately synchronous with or somenhat antecedent to the rise in agglutinin titre for the infecting

bacillus, and subsequently followed by the usual fall.

In this connexion it is useful to note that paratyphoid $B$ infection is particularly often found to be the occasion of a marked rise in the typhoid agglutinins. Further, such a rise in the typhoid agglutinins is often - especially in persons inoculated only against $B$ typho. sus-the first evidence presented of what subsequently proves to be paratyphoid B (or paratyphoid A) infection.

Rises of this kind in the agglutinin titre

of the serum for organisms other than the infecting agent have been attributed to the production of so-called co-agglutinins. But without entering on a discussion of the true meaning of "co-agglutination," it may be stated that when standardised agglutinable cultures are employed experience proves that notable co-agglutination is not met with in the enteric group. And these subsidiary rises in agglutinin titre are almost certainly to be explained as due to a re-stimulation by infection with a closely allied organism of the mechanisms already trained by inoculation to produce particular series of agglutinins.

Similar rises in agglutinin titre can be produced in im munised animals by the introduction of wholly heterologous bacteria. ${ }^{5} \quad$ But to what precise degree the same fact may apply to the effect of pyrexial attacks occurring in inoculated human subjects from causes other than enteric infections we do not at present know with certainty. Accordingly a diagnosis of typhoid or paratyphoid fever should not be based on a rise in titre of only 100 or 200 per cent., if it is clear that the maximum of that rise falls markedly outside the period of expectation for enteric fever ${ }^{10}$ (see 4, below). Yet it must be stated that the rises in enteric agglutinin titre so far met with in association with other febrile conditions very rarely amount to as much as 100 per cent., and are much more frequently either altogether absent, or so small as to be practically negligible.
4. The maximum of the curve of specific agglutinin formation accompanying enteric infection in man occurs between the sixteenth and the twenty-fourth day of the disease, and most frequently from the eighteenth to the twentieth day. And, so far as experience at present goes, a change of titre of even only 100 or 200 per cent., proceeding regularly in the usual curve, and passing its maximum within the period named, justifies a diagnosis of enteric fever. ${ }^{10}$ It will, of course, most commonly be found that the maximum has occurred in the interval between two successive observations.

5. In the case of mixed infections with enteric group bacilli (typhoid and paratyphoid), whether in inoculated or non-inoculated persons, the agglutinin curves for the different infecting organisms pursue their course independently of each other. They are therefore usually not synchronous, though they may be so.

Readings. - In readiog the agglutination tubes put up with different dilutions of serum what is looked for first is a tube exhibiting standard agglutination-that is, marked agglutination (naked-eye) without sedimentation (Dreyer); and the reading proceeds as described in the Directions for the Use of Standardised Agglutinable Cultures issued on behalf of the Medical Research Committee. Such readings give results entirely adequate for diagnostic purposes, if from three to five successive observations be carried out at intervals of from three to five days. Should a more precise determination of the limits of agglutination be required, as in experimental work or for the purpose of obtaining smooth and accurate curves, it can be obtained by reducing the intervals between the successive dilutions of the serum as described in the "Directions," or by attaching appropriately calculated values to various degrees of agglutination recognisable as increasingly greater and increasingly less respectively than standard agglutination. Thus Dreyer has shown that naked-eye differences in agglutination, ranging on each side of standard agglutination from total agglntination on the one hand to nil on the other, can be recognised distinctly when the serum dilutions increase or diminish in successive tubes by about 13 per cent., starting from standard agglutination as the central point and ranging upwards and downwards.

In the curves now to be described all the readings are expressed in standard agglutinin units (Dreyer) as defined in the "Directions," where the simple process of reducing an agglutination result observed in serum dilutions to standard agglutinin units per c.c. of serum is fully explained. The matter need not be described in detail here since the "Directions" are readily accessible in this country and have also been published in an article by W. C. Davison in the Journal of the Amerioan Medical Association (vol. Ixvi. (1916), pp. 1297-1300). The tubes were read in the usual manner after two hours in the water-bath at $50^{\circ}$ to $55^{\circ} \mathrm{C}$., followed by 15 minutes at the room temperature. Following Dreyer's full method, they were always read again for control after 24 hours' standing at room temperature, by which time any possible "zones of inhibition" have ceased 
to complicate the readings. The readings move on slightly in the 24 hours.

On each chart is given a table of the actual readings made. These readings are plotted out and curved dotted lines are drawn through the points thus obtained so as to indicate approximately the probable form of the agglutinin curve. Points between which a maximum is believed to lie are not joined, but the curve is there left open. Upon each chart is also entered the character of the protective inoculation which the patient had received (typhoid vaccine T., triple vaccine T.A.B.) and the diagnosis given by the agglutination tests is recorded. The letters T.A.B. stand throughout for typhoid, paratyphoid A, and paratyphoid $B$ respectively.

\section{Cases of Typhoid Fever.}

Chart 2.-A case of typhoid fever in a T.-inoculated individual. The T. agglutinin titre rises to more than double in the first four days. At the third observation, when the titre is probably already falling rapidly, it is 200 per cent. above the first reading. Maximum between twenty-first and twentyfourth day of disease. Reaction moderate, probably a mild attack. No "co-agglutination" for A or B accompanies the reaction. Clinically, irregular pyrexia, rising at intervals to between $102^{\circ}$ and $103^{\circ} \mathrm{F}$.

Chart 3.-Typhoid fever in a T.A.B.-inoculated individual. The measured rise in T. agglutinin titre is five-fold ( 400 per cent.) within nine days ; maximum between eighteenth and twenty-first day. The fall is to less than one-half in three days and to one-third in six days. From the readings in this case (as in Chart 2) we learn that the curve of rise is steeper than the curve of subsequent fall. The sympathetic rise and fall in $A$ and $B$ "inoculation" agglutinins are marked definite and approxımately synchronous. Clinically, temperature high at first $\left(103^{\circ}\right.$ F.) ; never above $99^{\circ}$ after fifteenth day.

Chart 4.-Typhoid fever in a T.-inoculated individual. This case again illustrates the great relative steepness of the rise in its later part as the maximum (probably sixteenth or seventeenth day) is approached. Its chief interest is in the existence of $A$ and $B$ agglutination curves running side by side, their maximum being pretty clearly a little antecedent to the T. maximum. The patient came from the Mediterranean area and gave a long history of diarrhoea, fever, $\& c$., before his removal to England as a convalescent. The present attack began three months later. His agglutination readings clearly show that he had suffered from mixed or consecutive $A$ and $B$ infection at some period previous to the present observations. Clinically, onset with shivering and headache. Three smart rises of temperature to $105^{\circ}$ $103.5^{\circ}$, and $102.5^{\circ} \mathrm{F}$. respectively on the first, third, and fifth day of illness simulating tertian ague. No malarial parasites in the blood. From the fifth day onwards temperature subnormal and rising to normal.

$$
\text { Cases of Paratyphoid A F'ever. }
$$

Chart 5.--Paratyphoid A fever in a T.-moculated individual. Reaction mild, but A agglutination rises in five days from less than 2 units to over 27 units (about 14-fold), a rise, be it noted, of at least some 1300 per cent. 'The fall is fairly rapid, but nothing like so steep. No " co-agglutination" for B. Sympathetic rise of T. inoculation-agglutinins marked and apparently pretty nearly synchronous. Clinically, very mild illness, temperature not exceeding $99^{\circ} \mathrm{F}$.

Chart 6.-Paratyphoid A fever in a T.A.B.-inoculated individual. Clinically jaundice, cholecystitis, severe and continued remittent and irregular pyrexia. The diagnosis paratyphoid $\mathrm{A}$ is obvious. The accompanying T. and B aggluti nation are shown on a large scale. They present points of interest and deserve examination.

$$
\text { Cases of Paratyphoid B Fever. }
$$

Chart 7.-Paratyphoid B fever in a $\mathrm{T}$-inoculated individual. This case is one already quoted (from Dreyer and Torrens) in a previous article. ${ }^{9}$ The $B$ agglutinins increase nineteenfold in eight days. Maximum between the eigh teenth and twenty-second day. The rise in $\mathbf{T}$. inoculation-agglutinins is marked and approximately synchronous. No co-agglutination of $\mathrm{A}$.

Chart 8.-Paratyphoid B fever in a T.-inoculated individual. A mild reaction, but the rise in $B$ agglutinins is at least nineteenfold. Maximum nineteenth or twentieth day of the disease. Maximum of sympathetic $T$. inoculation agglutinin rise probably antecedent. No co-agglutination of A. Clinically, diarrhoea and indisposition. The temperature never reached $100^{\circ} \mathrm{F}$., and only three times exceeded $99^{\circ}$ (evenings of seventeenth, eighteenth, and nineteenth days).

$$
\text { Late Cases. }
$$

Many cases do not come under observation here until late in the disease. These are often somewhat difficult to

The pyrexia of enteric fever in inoculated persons is very often quite atypical, particularly in the mild attacks. interpret, particularly when the day of onset is unknown, as
is frequently the case, for example, in wounded subjects suffering from sepsis and pyrexia.

Chart 9.-Paratyphoid A fever in a T.A.B.-inoculated wounded individual. Observations begin late in the attack, but the T.A.B. agglutinin curves all indicate the existence of active reaction. The differential diagnosis rests on the fact that in the case of $A$ the maximum has occurred later, and the amplitude of the reaction appears to have been greater than in the case of $T$. and $B$. agglutinins.

Chart 10.-Paratyphoid B fever in a T.A.B.-inoculated individual wounded 43 days before the first day of observation. The $B$ agglutinins fall steeply to one-third in three days and to about one-fifth after six days. T. and A agglutinins fall relatively slowly. Clearly a case of paratyphoid B infection.

Mixed and Consecutive Enteric Infections.

Chart 11.- Mixed infection; paratyphoid $A$ and $B$. Clinical condition, "trench fever." Moderate pyrexia, temperature falling to subnormal and normal on and after twentieth day of illness. Agglutinin titre for A rises eightfold in three days, that for B nearly sevenfold, both thereafter falling to less than one twenty-fourth of the higher reading in the next five days. Agglutinin titre for $T$. shows only a slight sympathetic rise; maximum antecedent to that of $A$ and $B$ agglutinins.

Chart 12.-Consecutive infection; paratyphoid B succeeding typhoid fever. The temperature fell (from $102^{\circ} \mathrm{F}$.) to subnormal on the first day of observation. It rose again on the sixth day, becoming subnormal again on the tenth day. The agglutinin chart also shows that we are dealing with two independent reactions: first, the later part of a B. typhosus infection; and, secondly, a B infection in which the $B$ agglutinins rise 300 per cent. at the second reading with an accompanying sympathetic rise in A agglutinins Judging by the speed with which the $T$. curve becomes flat the fall in $T$. agglutinins is distinctly checked by the $B$ reaction.

Chart 13.-Looks a little complicated. The day of disease is reckoned from the day on which the patient was wounded. He had long-continued very irregular, remittent, and intermittent pyrexia. The first stage of the illness is clearly paratsphoid A fever. The A aggiutinins first rise, and then fall from the second observation to one-fifth in the course of eight days. The T. and B agglutinins move only moderately in sympathy. But on the twenty-sixth day of disease it is noted that instead of continuing their normal fall $T$. and $B$ agglutinins begin to rise again, followed then or later on by the A agglutinins. Something new is evidently happening. Owing to the extreme gravity of the illness and accompanying delirium no further blood specimen was received until the fortieth day. B agglutinins were then found to have undergone a marked rise of about 135 per cent., and thereafter fell sharply to less than one-seventh of the highest observed value. The rise in A agglatinins was apparently less than on the former occasion, and its second maximum, like that of the T. agglutinins, preceded the maximum of $B$. agglutinins. The later part of the curves therefore indicates a paratyphoid $B$ infection following the original paratyphoid A.

\section{Pyrexia " not Enteric."}

Chart 14.-Three cases of pyrexia, with a rise and fall in $T$. agglutinins, diagnosed as not enteric. The recorded change in titre is less than 100 per cent. in each case, and the maximum falls in each case before (though not always very long before) the sixteenth day as judged from the form of the curves. Two of these cases, $(a)$ and $(c)$, are typical of those which cause the greatest difficulty in serum diagnosis They are recorded as being the most marked of their kind that I persunally have yet met with. On their charts as they stand opinion might perhaps not unreasonably differ as to whether the diagnosis of enteric fever was properly excluded or not. In such cases, which fortunately do not seem to be of very frequent occurrence, all available collateral evidence, both clinical and pathological, may require to be taken into consideration. And it may be suggested that from the hygienic standpoint a case that remains persistently in doubt had better be regarded as potentially enteric.

Chart 15.-Four examples of the more usual findings in febrile conditions other than enteric fevers. Here the diagnosis "not enteric" is obvious.

The evidence brought forward in the charts which bave now been described shows plainly that when standardised agglutinable cultures are employed in successive quantitative determinations of the agglutinin titre of the serum little difficulty is to be apprehended in the diagnosis and differentiation of the enteric fevers. In those cases which come under examination by Dreyer's method within two to three weeks of the onset of the disease no difficulty at all is likely to be met. But attention should be drawn to the fact that the majority of these cases here recorded ( 8 out of the 13) 

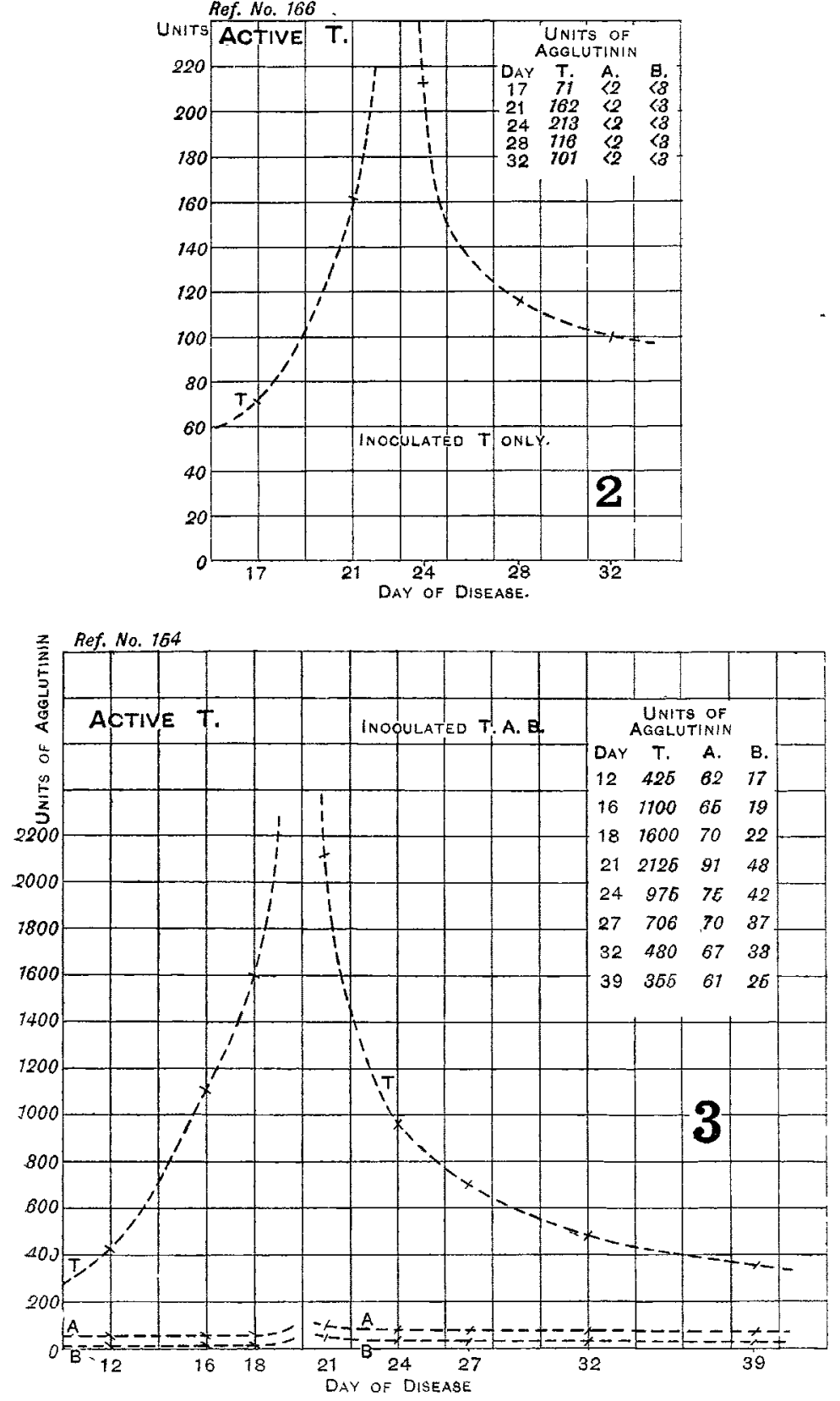
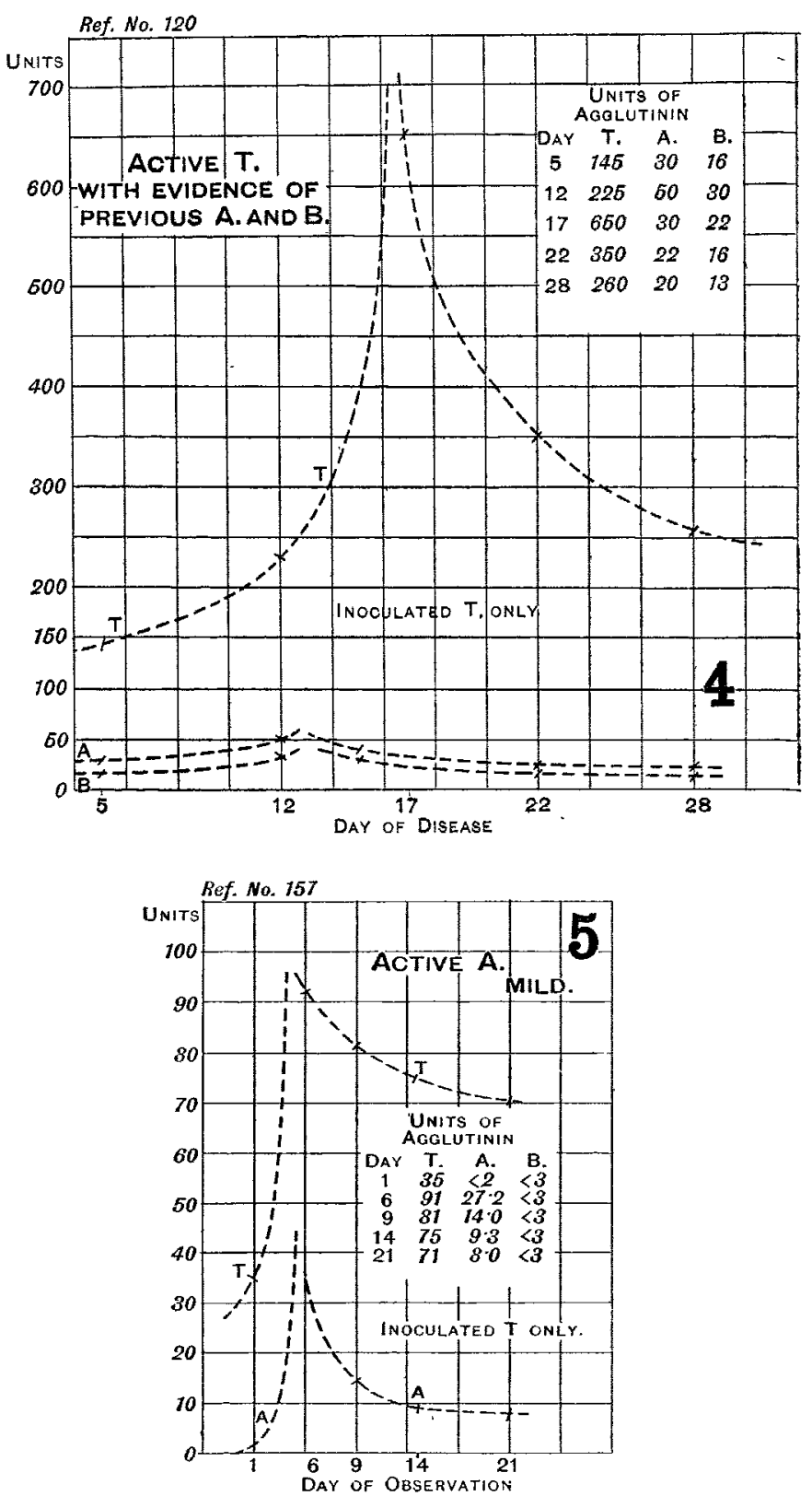
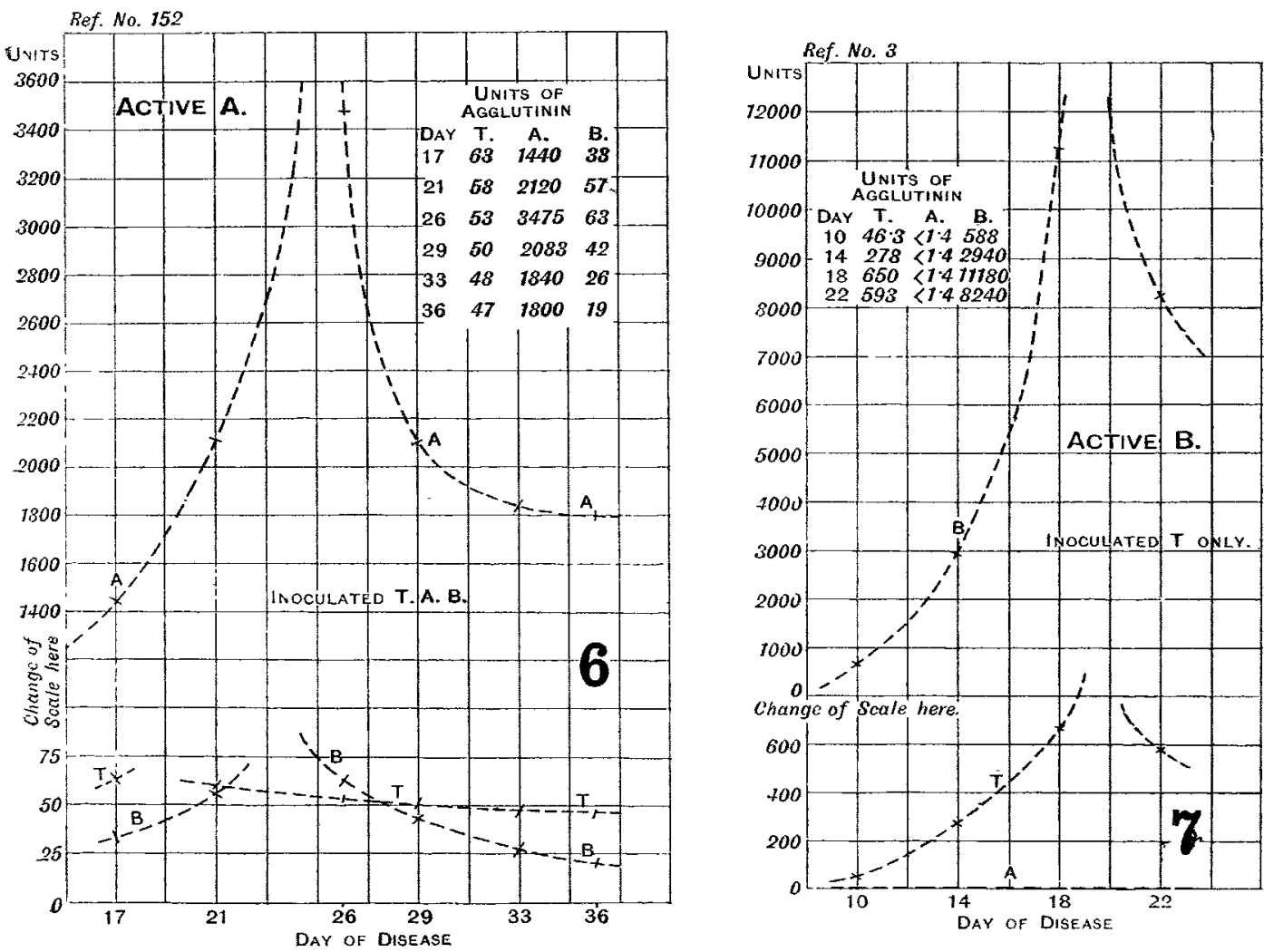

Ref. No 168

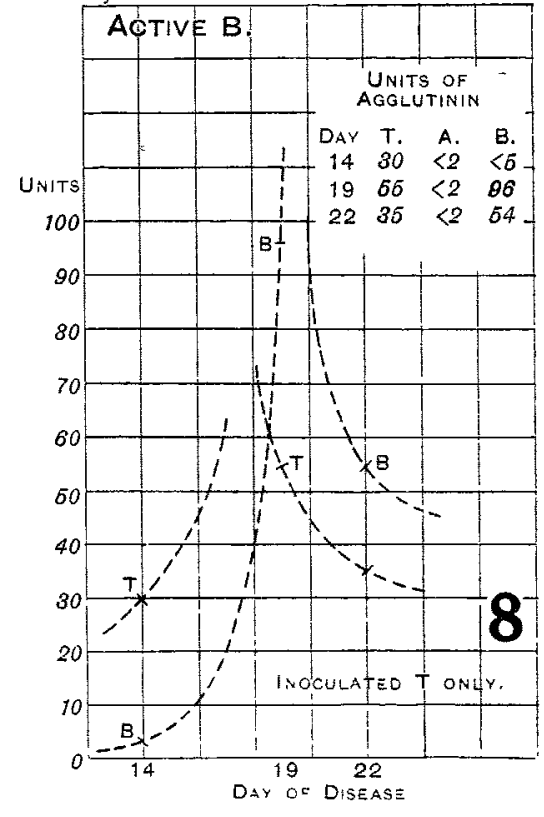



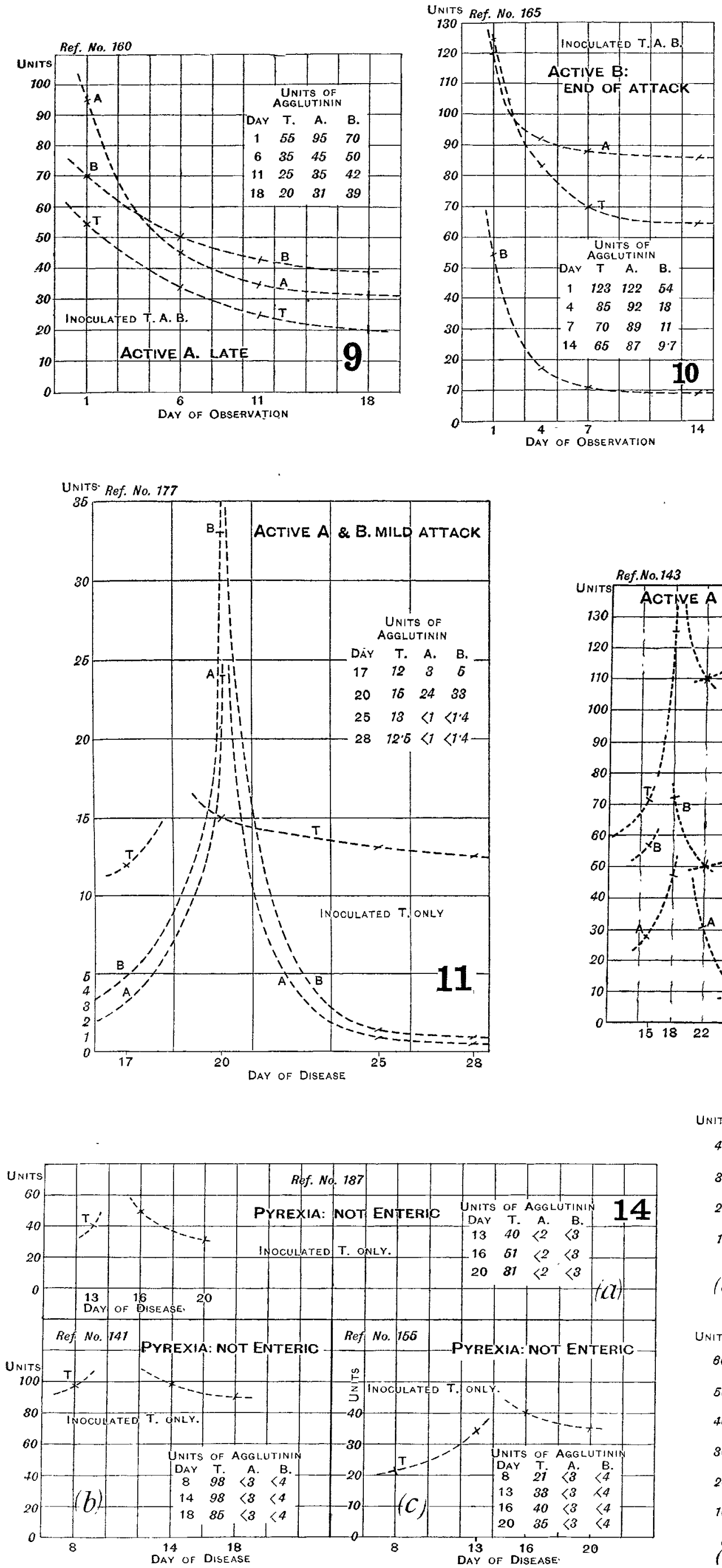
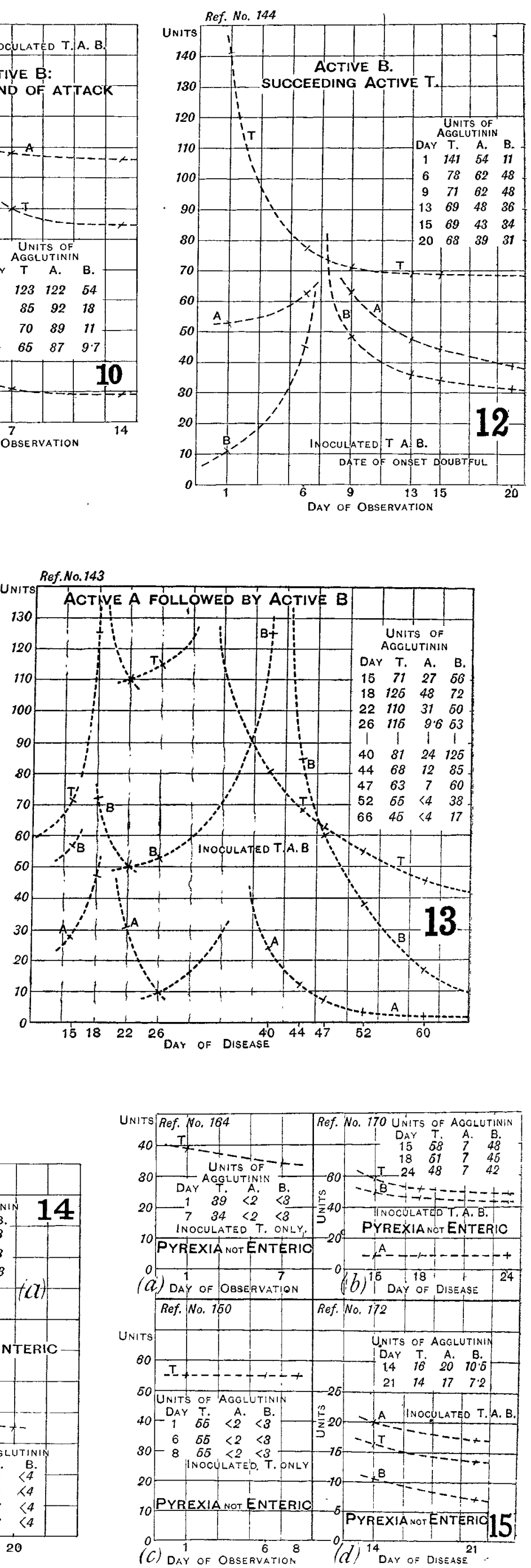
were what may fairly be spoken of as mild and atypical cases of enteric fever. For reasons already indicated elsewhere (Dreyer and Ainley Walker ${ }^{10}$ ) such cases are likely to occur with frequency under present conditions. It is, therefore, a matter of importance that this fact should be widely recognised, and that all doubtful febrile cases of whatever mildness should at an early period be submitted to agglutination tests before the possibility of enteric infection is excluded. If they are cases of enteric fever they will then be readily detected.

In a recent article, however, C. J. Martin and Upjohn conclude their communication with the statement that since the introduction of triple vaccine (typhoid, paratyphoid $\mathrm{A}$, and paratyphoid B) in the inoculation of Australian units "the interpretation of observations upon the agglutination of enteric organisms will ...... be too difficult to possess any practical value, and the isolation of the infecting organisms must be resorted to for diagnosis." The difficulty in the opinion of the present writer lies rather in persuading some observers to employ methods which have any "practical value" in inoculated persons. And the suggestion that reliance should be placed on culture methods finds its answer not only in the fact that with the most perfect technique and organisation methods of cultivation from blood, stools, and urine fail in quite half the cases which come under observation; but also in the statement made by Martin and Upjohn themselves that it would not have been possible for them "to have made more than one-fifth the number of observations by blood culture, and at least half of these would have miscarried as so many of the patients were past the first week of the disease on admission."

References.-1. Dreyer and Inman: The Lancer, July 31st, 1915. 2. Dawson, Sir Bertrand: The LANCET, March 20th, 1915, p. 602. 3 . Dreyer, Ainley Walker, and Gibson: TeE LANCET, Feb. 13th, 1915, p. 324. 4. Dreyer: Journal of Pathology and Bacteriology, 1909, vol. xiii., p. 332. 5. Dreyer : Proc. Roy. Soc. Me 1., 1915, vol. ix. (Medical Section), p. 9. 6. Madsen and Jörgensen: Festskrift ved Indv. af Statens Seruminst., Copenhagen, 1902. 7. Schroeder, K, Thesis, Copenhagen. 1909. 8. Dreyer and Ainley Walker: Journal of Pathology and Bacteriology, 1909, vol. xiii., p. 343 ; vol. xiv., p. 39.9 . Glynn and Cronin Lowe: The Lancet, Aug. 5th, 1916. 10. Dreyer and Ainley Walker : The LANCET, Sept. 2nd, 1916.

A PRELIMINARY NOTE ON

PIECES OF CLOTHING EMBEDDED IN
WAR WOUNDS.

BY R. J. WILlAN, M.V.O., M.S. DurH., F.R.C.S. ENG., STAFE SURGEON, R.N.V.R., ATTACEED TO R.N.H.S. "PLASSY", HONORARY ASSISTANT SURGEON, ROYAL VICTORIA INFIRMAKY, NEWCASTLE-ON-TYNEO

With a Note by Surgeon-General Sir W. WATSON CHEXNE, Bart., C.B., F.R.S., F.R.C.S. Eng.

I HAVE read with much interest Miss M. Davies's article in THE LANCET of Sept. 30th. For a considerable time I had been thinking over the question of the acute sepsis which is a sequel to the implantation of fragments of clothing in war wounds. and my interest was stimulated after the Jutland naval battle. Two lines of thought suggested themselves, viz., (1) that it would be a good thing if it could be known definitely if there was any foreign body, ordinarily non-transparent to $X$ rays, in the wound ; and (2) if the same foreign body could be treated with some antiseptic prior to its entry to the wound, and, if possible, that the same medium should be made to fulfil the two purposes.

\section{Jutland Naval Battle Patients with Shell Wounds.}

Twenty-six cases were received in my wards, which are classified under three groups: $(a)$ patients with lacerated wounds which were aseptic, $15 ;(b)$ patients with lacerated wounds which were septic, 6 ; and $(c)$ patients with both aseptic and septic wounds, 5. I regarded a wound as clinically aseptic when its lacerated margins were in a condition of dry gangrene, when it only required dressing once every two or three days, and when the patient's temperature and pulse-rate remained normal.

Of the above septic wounds either pieces of the wearer's clothing or boots were recovered from the wounds of 5 patients (i.e., 4 of Group $b$ and 1 of Group $c$ ). A marked feature of each septic wound was the offensive smell. Twenty of the patients had wounds which were clinically aseptic, while 11 of the cases had wounds which were septic. As the wounded were received nearly two days after the action no systematic search under general anæsthesia for fragments of clothing was made. Those obtained appeared in the ordinary process of dressing the wounds; it is quite possible that the other septic wounds also harboured similar foreign bodies in their depths.

The above figures are a good testimonial to the work done by the surgical staffs of the warships before transference to the hospital ship. They further show what a factor ordinary clothing plays in a wound becoming septis. This clothing must be saturated with pyogenic organisms derived from the exterior, also exuded from the wearer's skin.

In the trenches simple sterilisation by heat is insufficient, because the sterile clothing would be so quickly contaminated by the soldier's surroundings. If, therefore, any good is to accrue, the garments must be impregnated with some antiseptic; this would inhibit bacterial growth should a fragment be carried deep into the tissues by a piece of shell. Further, this antiseptic might prevent the rapid breeding of vermin, which is so bitterly complained of at the "Front."

\section{How can Clothing be made Opaque to $X$ Rays?}

Possibly this can be done by the recently described method of delineating the various internal soft tissues by $X$ rays. It can be done by the manufacturer treating the clothing and underclothing with a preparation of the constituent of indelible marking-ink. The prescription of this I have tried is : Dissolve in 3 ounces of liq. ammon. fort. $\frac{3}{4}$ oz. of nitrate of silver, and add $\frac{1}{4}$ oz. gum arabic and $\frac{1}{3}$ oz, archil. I did not soak the piece of cloth in the ink, but marked out on the cloth two groups of fine lines parallel to each other, the two groups being at right angles. It can be managed by periodically immersing the garments in some solution whose suspended particles are opaque to $X$ rays. As it is obviously desirable that the sailors' and soldiers' garments now in use could be utilised, the experiments were made with a substance that could not only throw a shadow with $X$ rays, but would also have some antiseptic properties.

\section{How can Clothing be made sterile?}

During the Russo-Japanese War the Japanese made an effort in their Navy to deal with the question by trying to make their crews don clean garments prior to an action. During the present war, British naval surgeons have done good work in persuading their ships' crews to wear clean, or even sterilised, clothing when an engagement is imminent. To attain an aseptic ideal it would be necessary to have sterilised clothing always ready when the ship is at sea; further, that the action suit is accessible. 'To be really accessible provision must be made for each man's clothing to be stored at the action station itself, in order that he could quickly effect a change when necessary. But to enable an aseptic ideal to be attained the State must provide a complete change of clothing for each man, just as they provide him during hostilities with a suit of warm clothing for the winter. The suit would then be under the absolute control of the responsible person in the ship, who would see that it was always ready sterilised for use. This aseptic ideal could be carried out in the Navy, but it would be useless in the trenches, where clothing is so quickly contaminated by the snrroundings.

In the Army the change of clothes could be kept at the bath house. On their way to the trenches the unit could change into their " trench clothes," and on their return from the trenches they could, after their bath, exchange in to their other suits. After the "trench clothes" have been baked, washed, and dried, they can be immersed in the antiseptic solution, and after drying they will be ready for a further spell in the trenches.

Instead of having the antiseptic in solution, perhaps it may be possible to have some volatile chemical which, when placed in the steriliser or baking chamber, would volatilise with heating and impregnate the clothing.

$$
\text { Experimental Work. }
$$

My experiments aimed at trying to find a single substance to treat the " action clothes" which would satisfy the following requirements: (1) would make the garments (or fragments of same) impermeable to $\mathrm{X}$ rays ; (2) would have some antiseptic properties; (3) would contain some antiseptic which was non-irritating to the tissues; (4) would contain some antiseptic which was non-irritating to the skin. I soaked 